\title{
El desarrollo profesional de los docentes de inglés en ejercicio: algunas consideraciones conceptuales para Colombia ${ }^{1}$
}

\section{In Service English Teachers' Professional Development: Some conceptual considerations for Colombia}

\author{
Melba Libia Cárdenas² \\ Adriana González 3 \\ José Aldemar Álvarez 4
}

\section{Resumen}

“Colombia Bilingüe” ha propiciado el crecimiento de programas de desarrollo profesional -PDP- para los docentes de inglés en ejercicio. En este artículo, los autores presentan conceptos básicos en el análisis de la formación continuada de los docentes; analizan los modelos de desarrollo profesional de Richards \& Farrell (2005) y James (2001); los autores también resumen los principales conceptos elaborados por académicos colombianos sobre el desarrollo profesional de los docentes de inglés, y proponen un marco conceptual para el país. Las conclusiones sugieren la inclusión de la estrategia del desarrollo y no la del entrenamiento, las perspectivas individual y social del aprendizaje y de la enseñanza, la teoría post-método, la creación de conocimiento en los contextos escolares y sociales y el aprendizaje y la práctica significativa del inglés entre los docentes.

Palabras clave:

Desarrollo profesional docente, enseñanza del inglés, investigación en el aula, conocimiento local, entrenamiento.

Bilingual Colombia has promoted the growth of professional development programs -PDP-for English teachers. In this article, the authors present some key concepts in the analysis of the continuing education of teachers; analyze the models of professional development proposed by Richards \& Farrell (2005) and James (2001); summarize the main concepts elaborated by Colombian scholars on the professional development of English teachers; and propose a conceptual framework for the country. Conclusions suggest that professional development programs should use teacher development and not teacher training; include the individual and social perspectives of learning and teaching; embrace post-method approaches; promote the creation of knowledge in school and social settings; and promote meaningful practice of English among teachers.

Key words:

Teacher professional development, english language teaching, classroom research, local knowledge, training.

Artículo recibido 24 de Febrero de 2010 y aprobado el 30 de abril de 2010

1 El Ministerio de Educación Nacional de Colombia -MEN-, en el marco del Programa Nacional de Bilingüismo, auspició en el año 2009 el "Estudio diagnóstico: oferta de programas de desarrollo profesional para los docentes de inglés en ejercicio en Colombia" (Contrato n. ${ }^{\circ} 867$ suscrito entre el Ministerio de Educación Nacional y ASOCOPI-Asociación Colombiana de Profesores de Inglés). Dicha investigación requirió, en primera instancia, la elaboración de un marco conceptual en dicho campo del que se deriva este artículo.

2 Profesora Universidad Nacional de Colombia, sede Bogotá. mlcardenasb@unal.edu.co

3 Profesora Universidad de Antioquia. agonzal@quimbaya.udea.edu.co

4 Profesor Universidad de la Salle. jalvarez@unisalle.edu.co 


\section{Introducción}

Siendo conocedores de los discursos profesionales locales e internacionales sobre qué debería abordarse en estos programas y cómo desarrollarse, podemos señalar que muy a pesar de contarse con esta amplia elaboración teórica e investigativa y con un creciente interés por parte de las universidades, en analizar el desarrollo profesional de los docentes de inglés en Colombia, no se ha abordado el tema del análisis de las ofertas de formación para docentes en ejercicio, en particular, de los marcos conceptuales que los motivan. Se espera que los postulados aquí presentados, así como un examen de los trabajos sistematizados sobre la base de experiencias realizadas en contextos nacionales colombianos, permitan avanzar en el conocimiento de los principios filosóficos que motivan dichos programas, en la relación entre aquellos y la ejecución de los mismos, y en la producción de nuevo conocimiento.

El área de formación permanente de docentes en servicio ha sido relativamente inexplorada en el campo de la lingüística aplicada (Richards \& Farrell 2005), aunque no se puede negar que se han dado pasos importantes en el estudio de las distintas necesidades de los docentes. En el caso de Colombia, este interés ha crecido notoriamente en los años posteriores al proyecto Colombian Framework for English-COFE- (Aparicio, Benavides, Cárdenas, Ochoa, Ospina y Zuluaga, 1995). Dicho proyecto resaltó la necesidad de atender no solamente la formación inicial de los docentes, sino de emprender programas de desarrollo profesional -PDP- para docentes de primaria y secundaria en ejercicio sobre la base de modelos internacionales y de las experiencias nacionales.

En la última década se han incrementado las oportunidades para acceder a programas que ofrecen diversas instituciones - principalmente universitarias. Paralelamente, y como resultado de los trabajos de investigación y de extensión de instituciones de educación superior colombianas, se han ido documentando experiencias que permiten vislumbrar una línea de pensamiento en el área (González, 1995, 2000, 2003, 2007; Cadavid et al., 2004; González y Quinchía, 2003; Clavijo et al., 2004; Mc Nulty y Quinchía, 2007; Gon- zález et al., 2001, 2002; Cárdenas, 2000, 2003, 2004, 2006, 2006a, 2008; Cárdenas y Nieto, 2007; Malagón y Cárdenas, 2007; Vergara, Hernández y Cárdenas, 2009; Álvarez, 2009). Este crecimiento en la oferta de programas está acompañado de la creación de conocimiento local y el desarrollo científico del país en el campo de la formación y desarrollo profesional, tal y como lo evidencia el aumento de publicaciones sobre esta temática en revistas arbitradas y eventos académicos nacionales e internacionales.

Por otra parte, se ha alcanzado más agenciamiento en el desarrollo de PDP en el marco del Programa Nacional de Bilingüismo -PNB- (Ministerio de Educación Nacional, 2007). Este programa ha permitido de manera especial la destinación de recursos públicos a la cualificación de los docentes de inglés (Usma, 2009). Es así como paulatinamente la formación del educador en servicio, se ha reconocido como un elemento primordial para la cualificación de procesos de enseñanza-aprendizaje del inglés como lengua extranjera.

Si se hace una mirada retrospectiva, se encuentra que desde los inicios de la lingüística aplicada, la formación inicial de los docentes y su cualificación en el ejercicio de la profesión ha recaído principalmente en aspectos de tipo metodológico, en los procesos de aprendizaje, y en general en cómo cualificar la enseñanza de una lengua extranjera. Dimensiones como la construcción del conocimiento profesional de base, su evolución, el papel de los programas de formación docente inicial y continuada en la construcción de dicho conocimiento, el proceso de crecimiento profesional y en general lo que se ha denominado el aprendizaje del profesor (teacher learning), aparecen como elementos poco abordados en estos programas. En esta misma línea de pensamiento surge una elaboración más compleja y reciente que se denomina: la cognición de los profesores (Woods, 1996). El estudio de la cognición de los profesores de lenguas se centra en lo que piensan, saben y creen y en la relación de estos aspectos con las prácticas de clase que llevan a cabo (Borg, 2006). Este crecimiento cognitivo ha recibido mayor atención en la formación inicial universitaria. Sin embargo, vale la pena preguntar si los aspectos 
señalados por Borg son igualmente importantes en los procesos de desarrollo profesional.

Teniendo en cuenta lo anterior, el presente artículo inicia con el examen y descripción del concepto de desarrollo profesional en profesores de lengua extranjera. Continuamos con una caracterización de las distintas propuestas que se erigen como modelos para que los profesores en servicio realicen procesos de crecimiento profesional de manera formal o informal. Estos modelos se examinan a la luz de las racionalidades y a la visión epistemológica que subyace a su estructura. Finalmente, presentamos la necesidad de incluir la investigación y la innovación como soportes de las propuestas de formación de los docentes de inglés en ejercicio, para ser consecuentes con la definición de desarrollo profesional, con las concepciones epistemológicas de los modelos y responder a las necesidades particulares de sus contextos.

\section{Conceptualizaciones básicas}

Para abordar el tema del desarrollo profesional de los docentes de inglés, que se encuentran en ejercicio en Colombia, consideramos importante definir algunos conceptos claves tales como: los objetivos de la enseñanza, el papel del docente, el aprendizaje del docente, las estrategias de formación de docentes y el desarrollo profesional de los docentes en ejercicio. $\mathrm{Si}$ bien estos conceptos son claramente diferenciables establecen entre sí, una compleja relación cuando se aborda el crecimiento de los profesionales de la enseñanza del inglés.

Kumaravadivelu (2003, p. 6) define como objetivo de la enseñanza "el crear las condiciones óptimas para que el aprendizaje deseable pueda tener lugar en el menor tiempo posible" (traducción propia). El autor opina que todo el andamiaje de la educación se basa en la idea de que la enseñanza realmente influye en el aprendizaje, a pesar de que no todo acto de enseñanza genera un acto de aprendizaje y de que existe un aprendizaje que no es el fruto de la enseñanza.

Independientemente de la relación establecida entre la enseñanza y el aprendizaje, la mayor parte de los análisis sobre la enseñanza se basan en la indagación sobre el docente. Kumaravadivelu
(2003) resume algunas concepciones que se han dado históricamente sobre el papel del docente en la enseñanza: técnico pasivo, practicante reflexivo e intelectual-trasformador.

En el primer papel se ubica al docente como un consumidor pasivo de conocimiento, producido por expertos y que lleva a cabo una serie de actividades prescritas para lograr los resultados del aprendizaje. Su trabajo es básicamente disciplinar, por lo tanto, su formación se hace en aspectos muy puntuales. En el segundo papel, el docente tiene además del conocimiento disciplinar, el conocimiento personal y la posibilidad de hacer investigación en el aula para mejorar su trabajo. En el tercer papel, el más reciente de todos, el docente reúne los dos anteriores, y los enriquece con el desarrollo de su conciencia sociopolítica y una visión holística de la enseñanza que ubica su trabajo en la sociedad y no solamente en el aula o la escuela.

La formación de los docentes tiene como objetivo central la adquisición de aprendizajes para desempeñarse en la labor de la enseñanza. Aunque existe un consenso sobre ¿qué es lo que deben aprender los docentes? (conocimiento profesional o conocimiento base [Shulman, 1987; Freeman \& Johnson, 1998; Gatbonton, 1999; Johnston \& Goettsch, 2000; Mullock, 2006]), el estudio científico -sobre cómo aprenden los maestros- es muy reciente. En este sentido es indispensable ubicar el aprendizaje de los docentes dentro de los principios generales de la andragogía (Knowles, 1984). En relación con el aprendizaje en adultos Pont y otros (2003, p. 165)] sustentan que para que éste sea efectivo y exitoso, debe:

- Ser motivante, es decir, que sus objetivos deben ser precisos.

- Tener objetivos que puedan ser realmente alcanzados por las personas y que representen un reto que no sea imposible.

- Requerir que el maestro se apoye en el conocimiento adquirido previamente para que no se sienta perdido y para que, al mismo tiempo, lo pueda potenciar y él pueda tener la sensación de poder utilizar su conocimiento en la vida diaria. 
- Dar a la persona la oportunidad de elegir para que pueda ejercer su nueva autonomía.

- Desarrollarse en un periodo de tiempo suficiente para que el maestro pueda estar a cargo de sus demás obligaciones y ocupaciones.

- Conducirlo a un logro final y ayudarlo a plantear cómo alcanzar los objetivos propuestos inicialmente.

Aprender a enseñar, se define por Freeman (1989) como el resultado de cuatro componentes: 1) 'el conocimiento', que incluye la disciplina que se enseña, a quién se enseña y dónde se enseña. 2) 'las habilidades', que incluyen los procedimientos de instrucción, manejo de clase y decisiones curriculares. 3) 'la actitud', que es la forma como el docente se concibe y concibe la enseñanza y la relación con los estudiantes. Y finalmente, 4) 'la conciencia, considerada como la capacidad de reconocer aspectos o hechos importantes del aprendizaje que motivan la toma de decisiones. Los dos primeros componentes se llaman: "conocimiento de base del docente", y se constituyen en los pilares de la mayor parte de los programas de formación.

Johnson (2009) dice: "Lo que sabemos del aprendizaje de los docentes cambia si se mira desde una perspectiva socio-cultural. El contenido, la estructura y los procesos de formación de los docentes entendidos como procesos socio-culturales deben ayudar a entender que "el desarrollo cognitivo no es un simple asunto de enculturación o de apropiación de los recursos y prácticas socioculturales, sino la reconstrucción y transformación de dichos recursos y prácticas de manera que respondan a las necesidades individuales y locales'"' $(2009$, p.2).

Dentro de la formación que tienen los docentes para aprender a enseñar, se pueden ubicar dos estrategias mayores: el entrenamiento y el desarrollo (Freeman, 1989). Es importante aclarar que estas dos estrategias aplican tanto a la educación formal del docente en la universidad (o también llamado "pre-service", es decir, anterior a su ejercicio docente) como a la actividad laboral posterior a esta formación inicial (o "in-service”).
Freeman (1989) define el entrenamiento para profesores como un tipo de relación entre el docente y el formador, facilitador, guía o colaborador, en el que este último interviene directamente en la forma como el docente enseña. Esta intervención se basa en el hecho de considerar que se pueden conseguir mejores resultados del proceso de enseñanza "a través de una clara secuencia de pasos, dentro de un período específico de tiempo". Estos aspectos de la enseñanza que se ven como "susceptibles de ser entrenados" son unidades diferenciadas, generalmente basadas en conocimientos o habilidades que pueden ser aisladas, practicadas, y, finalmente, dominadas" (1989, p. 39, traducción propia). Es así como la enseñanza se evalúa por medio de criterios observables que se alcanzan en determinado tiempo. El entrenamiento es una estrategia de intervención clara y directa que asume el éxito de la actividad docente en el dominio de estas habilidades y comportamientos. Aunque esta estrategia puede ser efectiva, a menudo es una visión fragmentada y de corto impacto de la enseñanza.

Para el caso del entrenamiento docente, Aparicio y otros $(1995$, p.3) afirman que éste "se refiere a las actividades directamente enfocadas a las responsabilidades presentes del docente y usualmente tiene objetivos a corto plazo y metas inmediatas" (traducción propia). Por otro lado, continúan los autores diciendo que "el entrenamiento involucra el entendimiento de conceptos básicos y principios, y la adquisición de habilidades instruccionales son pre requisitos para el desempeño en el salón de clase. Merece resaltarse que los contenidos de un proceso de entrenamiento son en general determinados por expertos, quienes elaboran formatos o documentos estandarizados sobre la labor pedagógica en los que se prescriben las actividades y roles; en pocas palabras, el accionar pedagógico. Éstos se acompañan generalmente de procedimientos puntuales que deben seguirse paso a paso para lograr los beneficios que traen consigo estos programas. Es decir, que se utilizan recetas pedagógicas pre empaquetadas para ser seguidas por los educadores-usuarios. Adicionalmente, Aparicio y otros (1995) advierten que debido a su focalización hacia lo instrumental, 
este modelo ha tenido usualmente como ejes de entrenamiento el conocimiento de la materia en sí, en nuestro caso el inglés, así como también los niveles de la lengua y sus habilidades.

De manera similar se ha enfatizado el desarrollo de habilidades para la enseñanza, es decir, el componente metodológico. Es así como algunas de las temáticas propias de este modelo son el manejo de la disciplina en la clase, los materiales de instrucción, las estrategias, procedimientos y técnicas. Este modelo descomplejiza el proceso de formación y de enseñanza-aprendizaje al promulgar una filosofía en la cual se infiere la adquisición de conocimientos pre empacados y ofrecidos en un periodo de tiempo corto, bajo la ilusoria verdad de desarrollo profesional. No obstante, se ignoran aspectos tales como la continuidad del proceso, la reflexión y la conexión entre los contextos de los docentes, sus construcciones pedagógicas y la teoría que se promulga en tales cursos.

El entrenamiento docente, de acuerdo con Narváez (2005, p. 247), concibe la formación como "un asunto estático y referido sólo al dominio de las destrezas conceptuales". Bajo esta perspectiva, los maestros son tratados como receptores de modelos y herramientas para su aplicación de manera que lleven al crecimiento profesional y a su eficiencia pedagógica. Este enfoque se caracteriza por asumir una concepción instrumentalizante que se sustenta en el conductismo y el tecnicismo en el que, como dice la autora, se le enseña al maestro a enseñar sin orientarlo hacia tensiones conceptuales ni experienciales que le permitan reevaluar su rol en la escuela y en su profesión.

En el marco de este primer enfoque, los programas de formación profesional continuada se fundamentan en el principio de la corrección de las desviaciones instruccionales (Díaz-Maggioli, 2003a) provenientes de una mala formación en el pregrado. Es una visión correctiva en la cual el docente llega con prácticas pedagógicas inapropiadas, las cuales deben ser erradicadas. En este enfoque se articulan una racionalidad artesanal y técnica de la enseñanza, del currículo y de la formación del docente (Grundy, 1998; Castro et al., 2004). Imbernón (1997, p. 37) se refiere a este modelo como "orientación perennialista y racional técnica" por cuanto se centra, sobre todo, en la formación de los contenidos académicos que hay que transmitir, en el concepto de que saber es hacer, y en el conocimiento y la capacidad para aplicar ese conocimiento (es decir, 'poder'). Así la imitación de un modelo de enseñanza y la conceptualización del quehacer pedagógico como un conjunto de técnicas aplicables a cualquier contexto y población se constituyen como ejes de este enfoque.

Woodward (1991) establece que los programas de entrenamiento provienen de una relación asimétrica en la cual un ente externo coacciona para que los docentes participen en ellos. Además, el motor de dichos programas es el desarrollo de competencias o destrezas con el objeto de la adquisición de conocimientos. Su duración es limitada, lo que no permite el desarrollo de un debido proceso de crecimiento. La agenda que constituye estos programas se establece por sujetos, generalmente expertos, o entidades externas dejando de lado los intereses y necesidades de los maestros. Así mismo, los procesos que se desarrollan encuentran un horizonte en la consecución de un producto o un certificado más que la mirada sobre el proceso en sí. Los modelos de entrenamiento impositivos, se utilizan generalmente para la implementación de políticas lingüísticas educativas, desconociendo las realidades en las que se implementan, dejando por fuera la opinión de los docentes, actores educativos que las ejecutan, y apoyándose en agencias académicas de exámenes (Shohamy, 2006).

Por otro lado, definimos el desarrollo como una estrategia indirecta que tiene una mayor permanencia en el tiempo ya que su propósito es generar cambio a través del aumento o la modificación de la atención que prestamos de manera consciente a asuntos determinados (Freeman, 1989). Esta estrategia se concentra más en la actitud y la conciencia del docente y se lleva a cabo con la ayuda de un colaborador, también conocido en nuestro ámbito como tutor o formador de docentes. En consecuencia, el desarrollo depende altamente del docente como individuo, del papel del colaborador y de la interacción entre ellos. El colaborador utiliza estra- 
tegias para motivar la conciencia y comprensión del docente sobre una situación determinada, es decir, para cuestionarlo acerca de lo que este último está haciendo y por qué. Las soluciones las genera el docente con o sin la ayuda del colaborador, pues es el docente quien debe tomar las decisiones.

Una de las principales características del desarrollo es ser permanente y abierto en el tiempo, ya que es el docente quien define cuándo termina el trabajo. El desarrollo se encamina a buscar opciones pedagógicas para la trasformación y construcción del saber y quehacer pedagógico basado en la reflexión sobre la praxis. En contraste, el concepto de desarrollo docente comprende el crecimiento integral que no se limita a una tarea o elemento especifico de la enseñanza. Éste se propone metas a largo plazo y se empeña en facilitar el progreso de los docentes con respecto a la comprensión de la enseñanza y de ellos mismos como educadores (Richards \& Farrell, 2005). El desarrollo profesional puede ser iniciado en parte o totalmente por los docentes mismos, haciéndose así más individualizado y flexible (James, 2001). Diferente al entrenamiento, más que la prescripción, el modelo del desarrollo docente, busca la descripción, la comprensión y el entendimiento de las diferentes dimensiones de las prácticas pedagógicas.

El concepto de desarrollo se articula con el ejercicio de la reflexión pedagógica (Wallace, 1991; Richards \& Lockhart, 1994; Freeman, 1989, 1998; Schön, 1987), lo cual permite agenciar y posicionar al sujeto como centro, superando la visión del entrenamiento en la cual el objeto, el conocimiento estático de la materia o área de conocimiento, se superpone opacando los procesos de análisis crítico, la construcción de teorías y reflexión desde la experiencia por parte del individuo. El proceso de desarrollo avoca por un enfoque desde-abajo (bottom-up), en contraposición a un enfoque desdearriba (top-down) predominante en los modelos de entrenamiento (James, 2001).

En la actualidad el desarrollo profesional es la estrategia más aceptada, pero esto no implica que sea la más utilizada. Aunque la participación activa del docente constituye el deber-ser del desarrollo docente, algunos autores presentan miradas pesi- mistas frente a estos programas como lo explican Dede et al. (2009), Loeb et al. (2007), González (2009) y Díaz-Maggioli (2003a). Por ejemplo, este último autor afirma que incluso algunos programas de formación permanente o crecimiento profesional se han adherido a la filosofía de "en-una-medidaencaja-todo", es decir, un enfoque desde-arriba, de manera que algunas iniciativas se han convertido en "paquetes de reforma" con decisiones acerca de qué, cuándo y cómo los docentes deben aprender algo. En consecuencia, el concepto de crecimiento profesional puede llegar a perder validez para convertirse en un proceso más de entrenamiento si no involucra a los docentes a lo largo de la planeación y todo su desarrollo.

Al considerar que el aprendizaje sobre la enseñanza es un proceso no acabado, se acepta que los docentes en ejercicio requieren oportunidades para mejorar su conocimiento y habilidades de enseñanza. En este sentido, se habla de desarrollo profesional. Díaz-Maggioli (2003b, p. 1) define el desarrollo profesional como un "proceso evolutivo de autorevelación, reflexión y crecimiento que arroja los mejores resultados cuando se sostiene en el tiempo en comunidades de práctica y se centra en las responsabilidades relacionadas con el trabajo" (traducción propia). El desarrollo profesional se refiere generalmente al crecimiento profesional "a largo plazo que busca facilitar el crecimiento de los docentes en su comprensión de la enseñanza y de ellos mismos como docentes. A menudo incluye el análisis de diferentes dimensiones de la práctica docente como base para una revisión reflexiva y puede ser por lo tanto iniciada desde la base" (Richards \& Farrell, 2005, p.4).

Díaz-Maggioli (2003b) señala que el desarrollo profesional de los docentes en ejercicio se hace necesario por razones tales como las reformas educativas y los nuevos retos que éstas imponen a los docentes; la relación entre la forma como enseñan los docentes y el éxito de los estudiantes; la necesidad de que los objetivos de la enseñanza se ajusten a las necesidades de los estudiantes; y en el caso de los docentes de lenguas extranjeras, que los docentes sean capaces de ayudar a sus estudiantes a desarrollar la competencia lingüística y cultural en 
la lengua extranjera que aprenden. Adicional a estas justificaciones, consideramos importante señalar que los docentes de inglés como lengua extranjera en Colombia han reconocido que una de las principales necesidades profesionales que enfrentan es su nivel lingüístico (González, et al., 2002), ya que éste tiende a verse afectado por la falta de práctica significativa, en especial entre los docentes de instituciones educativas públicas (González, 1995).

El desarrollo profesional de los docentes en ejercicio generalmente se da bajo la forma de cortos programas particulares a las instituciones escolares, talleres o cursos ofrecidos en los distritos escolares por universidades donde la asistencia es obligatoria o voluntaria. Generalmente son financiados por las instituciones educativas, las autoridades gubernamentales, los docentes mismos o las tres instancias. Los programas de desarrollo profesional apuntan a mejorar las condiciones del aprendizaje de los estudiantes y la calidad de la educación. En el caso de los docentes de inglés en Colombia, González (2003) encontró que el desarrollo profesional de los docentes en ejercicio se basaba principalmente en los congresos profesionales locales y nacionales y las charlas académicas organizadas por las editoriales que venden libros de enseñanza del inglés. En dicho estudio se mencionaba la poca oferta existente por parte de las universidades y la falta de apoyo de los entes gubernamentales locales para la financiación de programas pertinentes a las necesidades de los docentes.

Desimone, Smith \& Ueno (2006) concluyen, luego de una revisión de experiencias sobre desarrollo profesional de docentes en ejercicio, que las mejores prácticas al respecto se caracterizan por: tener mayor contacto entre los formadores y los docentes; desarrollar actividades en períodos más largos de tiempo; incluir docentes del mismo contexto educativo; dar variadas oportunidades para el aprendizaje activo; ser coherentes con las reformas educativas; y centrarse en el desarrollo del saber específico. Los autores dicen que, en particular, "el desarrollo profesional que es sostenible en el tiempo y que se centra en contenido académico ha surgido tal vez como la forma más importante de formación para los docentes en ejercicio" (2006, p. 182).

\section{Paradigmas actuales del desarrollo profesional de los docentes de inglés en ejercicio}

A la luz del análisis de la estrategia del desarrollo profesional (Freeman, 1989), como la mejor alternativa para comprender la formación inicial y permanente de los docentes, es posible determinar que esta estrategia supera el paradigma transmisionista e instrumentalista de la racionalidad artesanal y técnica del modelo de entrenamiento. Esta concepción crítica se fundamenta en la experiencia y creatividad del profesor para afrontar y desarrollar escenarios claramente identificados en el contexto, en el que la reflexión y cuestionamiento de creencias, valores y prácticas son una de las fuentes de crecimiento. Crookes (2009) insiste en la necesidad de que los valores, creencias y filosofías personales y colectivas de los docentes sean tenidos en cuenta no sólo como fuentes de datos en investigaciones, sino como parte fundamental de la construcción de la profesión docente en cualquier programa de formación. De igual manera, Johnson (2009) dice que "la formación de los docentes debe estar orientada por un cambio de paradigma epistemológico, reconociendo la naturaleza eminentemente social del aprendizaje y de la enseñanza". La autora afirma que en una perspectiva socio-cultural, aprender a enseñar se basa en la idea "de que saber, pensar y comprender vienen de la participación en las prácticas sociales de aprendizaje y de enseñanza en situaciones escolares y de aula específicas" (2009, p. 13). Adicionalmente, estas racionalidades sitúan al docente en el ámbito de la investigación en el aula, como planificador de decisiones, reflexivo y con capacidad de vincular su área de enseñanza con la formación, actualización y conciencia histórica de la realidad en donde emerge (Stenhouse, 1984, 1987; Kemmis \& Mc Taggart, 1989).

El desarrollo profesional ha sido caracterizado dentro de un paradigma práctico-crítico por varios autores como Woodward (1991); Wallace (1991); Aparicio et al. (1995); Freeman (1989, 1998); Cárdenas (2000, 2003, 2004); Cárdenas \& Malagón (2007); Cárdenas \& Nieto (2007); Díaz-Maggioli (2003b); Richards \& Farrell (2005); y Narváez (2005), entre otros. Haciendo un análisis de estos postulados 
podemos afirman que las condiciones para el desarrollo profesional de este paradigma deberían ser las siguientes:

1. Programas basados en procesos de reflexión sobre la práctica en los cuales se integre a los docentes y se estructuren con base en sus necesidades e intereses. En otras palabras, la agenda de estos programas debe ser interna más que externa a lo que los docentes mismos buscan.

2. La orientación sobre el crecimiento, más que sobre la adquisición de herramientas instruccionales momentáneas, permite la integración del conocimiento que los docentes traen consigo como producto de su experiencia. En este sentido, los programas que permanecen en el tiempo son más efectivos.

3. Programas en donde la construcción sea colectiva y colegiada, de manera que el diálogo y la colaboración entre los integrantes del proceso pedagógico permitan el crecimiento profesional.

4. Un enfoque cuestionador en el cual los docentes indaguen alrededor de cualquier problemática educativa que les permita establecer ciclos de reflexión y experimentación con nuevas ideas para que después puedan adaptar a sus contextos educativos.

5. El establecimiento de sistemas de apoyo sostenidos en el tiempo serán útiles para que obtengan más impacto en los docentes y en las prácticas que realizan en las poblaciones con las que están en contacto directo.

6. La construcción de escenarios en donde la práctica y la teoría entren en diálogo, a partir de las experiencias y la praxis de los docentes. Estos programas ofrecen la posibilidad de compartir experiencias, formar grupos de interés y construir una comunidad académica alrededor de las problemáticas educativas.

7. La investigación acción como principal componente de los programas de formación docente, lo cual permite la generación de nuevo conocimiento pedagógico, su transformación o actualización y el impacto en las comunidades educativas en las cuales se desempeñan los docentes.

8. La estimulación de la autonomía de los maestros en dimensiones tales como la afectiva, la intelectual, la cultural y la social. De esta manera, se rescata la figura del maestro como un sujeto pensante, con capacidad para tomar sus propias decisiones en vista de su conocimiento y capacidad reflexiva frente a sus contextos de enseñanza.

9. La dimensión volitiva es primordial en el desarrollo de programas de crecimiento profesional. Un principio básico para su éxito es la disposición del docente para participar en éstos, y no que sea el resultado de una imposición externa.

10. Las actividades y su evaluación en el marco de estos programas deben estructurarse con una visión orientada hacia el proceso, más que hacia los productos provenientes de ellas.

11. Una de las principales características del desarrollo profesional debe ser el de la superación del paradigma del experto que presenta modelos para ser imitados por los profesores con el fin de darle cabida al colega que está más cercano a la experiencia de los otros, y quien es una fuente primaria de enriquecimiento y crecimiento. En esta perspectiva, la imitación puede surgir de manera voluntaria. Este concepto es consecuente con posturas epistemológicas como la teoría post método (Kumaravadivelu, 1994; 2003) en sus principios de particularidad, posibilidad y practicidad. Estos principios hablan de la necesidad de tener programas especiales para cada contexto, de ayudar a los individuos a hacer posible lo que quieren y de construir formas prácticas, derivadas de teorías determinadas.

\section{Aproximaciones teóricas al desarrollo profesional de los docentes en ejercicio}

Consecuentes con las características del desarrollo profesional de los docentes en ejercicio, dentro de la 
concepción de desarrollo, hemos elegido dos modelos dinámicos que representan una adecuada conceptualización para nuestro contexto: los modelos de Richards \& Farrell (2005) y el de James (2001).

\subsection{La propuesta de Richard \& Farrell (2005)}

La concepción de los autores sobre el desarrollo profesional de los docentes en ejercicio se resume en la Gráfica 1:

Gráfica 1. El desarrollo profesional, según Richards \& Farrell (2005).

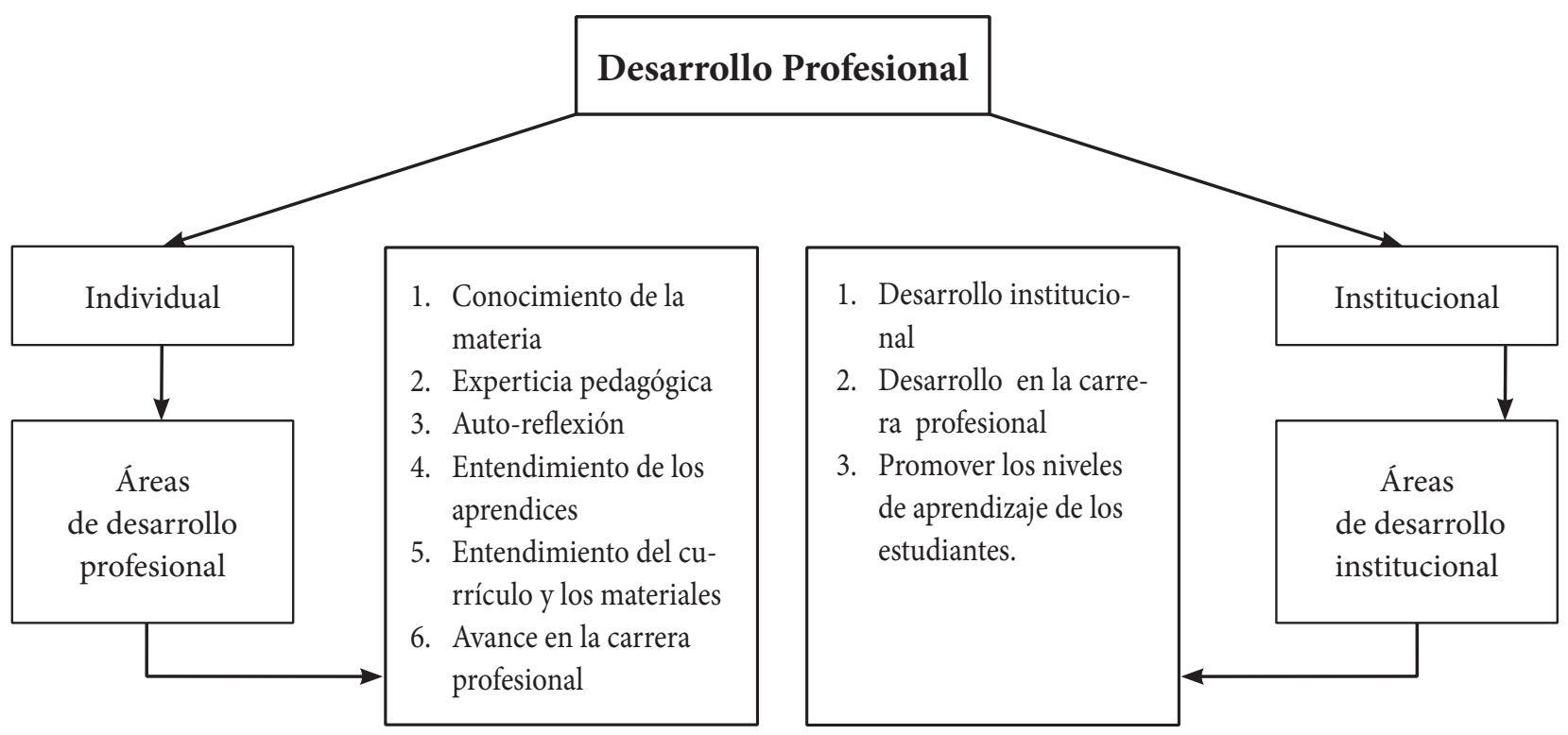

Obsérvese que este modelo presenta dos dimensiones: una en el ámbito de lo individual y la otra en lo institucional. En relación con lo institucional es justo precisar que usualmente la intención de una institución al promover PDP para sus docentes está ligada a la mejoría de la entidad en los tres dominios que se esbozan en la gráfica: el desarrollo de la institución, de los docentes y de los estudiantes. La inserción del segundo dominio inmediatamente establece una conexión con la primera dimensión: la individual, en la que las áreas de desarrollo profesional se centran en el docente, sin olvidar que existen los estudiantes (entendimiento de los aprendices) y una institución (entendimiento del currículo) representada en un currículo. Aunque no siempre las instituciones auspician la participación de sus docentes en PDP, la relación entre las dimensiones institución e individual es directa.

Así como en los modelos tradicionales, este modelo incluye elementos tales como lo metodológico y el saber disciplinar lingüístico. No obstante, éstos no

se constituyen en el motor de su desarrollo, y por el contario se complementan con elementos tales como la reflexión y el interés por la promoción en la carrera profesional dentro del marco de lo formal y de lo informal, puesto que cada instancia de desarrollo profesional es un momento más de crecimiento tanto en la escala personal como institucional. Detallamos a continuación los saberes que hacen parte de cada una de las citadas dimensiones, adaptándolos a la formación de docentes de inglés:

\section{La dimensión individual comprende:}

1. Conocimiento de la materia: el conocimiento disciplinario en aspectos como gramática del inglés, análisis del discurso, fonología, evaluación, adquisición de una segunda lengua, metodología, desarrollo del curriculo, y otras áreas que hacen parte del conocimiento base profesional.

2. Experticia pedagógica: conocimiento de nuevas áreas de la enseñanza, capacidad para 
enseñar en diferentes áreas de habilidades a aprendices de diferentes edades y experiencias.

3. Auto-reflexión: conciencia propia, es decir, conocimiento de uno mismo como un educador, de sus propios valores, principios, fortalezas y debilidades.

4. Entendimiento de los aprendices: profundización en la comprensión de los estudiantes, de sus estilos, problemas, dificultades, y de las formas de hacer el contenido más accesible a los estudiantes.

5. Entendimiento del currículo y de los materiales: profundización en el conocimiento de currículo y de alternativas curriculares, uso y desarrollo de materiales de instrucción.

6. Avance en la carrera profesional: adquisición de conocimientos y experiencias requeridas para el desarrollo personal y la promoción laboral.

Desde la dimensión institucional, se considera necesario que los docentes se actualicen debido a nuevas asignaciones académicas y cambios en el currículo. En consecuencia, el desarrollo docente está asociado a las necesidades de la institución. En este marco la institución busca que sus procesos educativos mejoren $y$, por correlación, hagan una contribución a la formación del docente.

\section{Los objetivos de esta perspectiva son:}

1. Desarrollo institucional: mejoría del desempeño de la institución educativa en términos del aprendizaje de los estudiantes y mejor posicionamiento en el mercado.

2. Desarrollo en la carrera docente: mediante una actualización profesional se busca facilitar la promoción de los docentes a otras posiciones dentro de la institución. Se busca así satisfacción laboral, la cual se ve reflejada en un mejor desempeño de los profesores y en una mayor estabilidad laboral.

3. Promover el nivel de aprendizaje de los estudiantes: se busca que los estudiantes aumenten sus niveles de desempeño en la institución, de manera que tanto la institución como sus profesores se proyecten bien hacia el exterior.

\subsection{La propuesta de James (2001).}

La propuesta de James (2001), sobre formación permanente para maestros se visualiza en la Gráfica 2:

Gráfica 2. El desarrollo profesional, según James (2001).

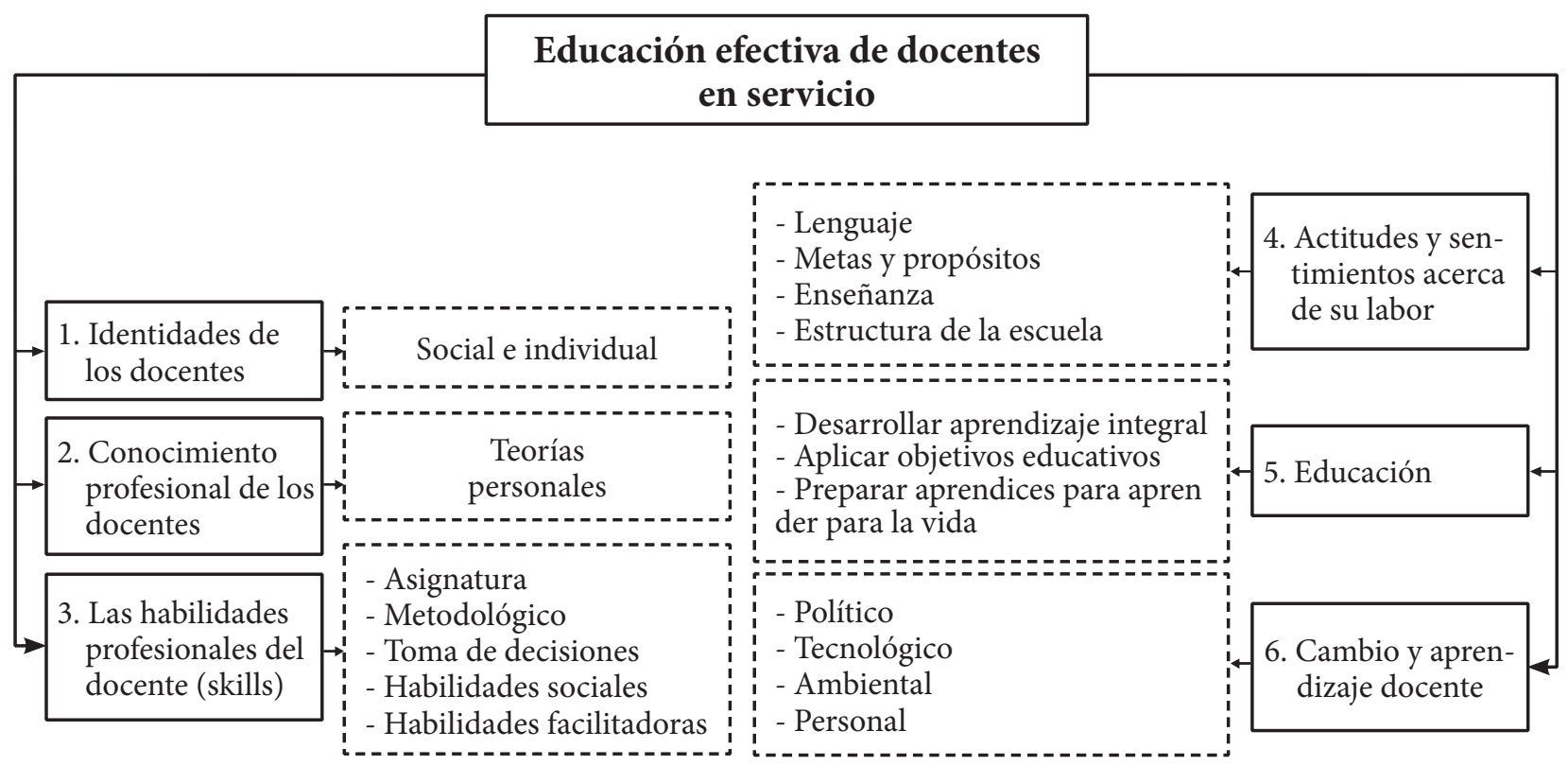


Esta propuesta presenta seis componentes que buscan desarrollar de forma holística en los docentes, un conocimiento profesional, habilidades $y$ actitudes. El autor centra este proceso en tres dimensiones: 1) personal $(1,4,6) ; 2)$ disciplinar $(2,3)$ y 3$)$ educacional (5). Algunos componentes tales como identidades docentes (1), educación (5), y el cambio y aprendizaje del docente (6), están estrechamente relacionadas con ámbitos sociales, igualmente identificados por Johnson (2009).

En el caso del primer componente de este modelo, se enfatiza en el hecho de que el maestro -como un ser social- establezca unas relaciones tripartitas interactivas en el aula: el maestro, los estudiantes y el currículo. Sin embargo, estas relaciones se ven impactadas por otros contextos que van más allá de los límites del salón de clase como la comunicad local, padres, otros docentes, supervisores, etc. También se pueden establecer conexiones con otras regiones y la comunidad internacional que mediante ciertas instituciones ejercen influencia en lo que pasa en un salón de clase.

El componente dos, conocimiento profesional de los docentes, se refiere a todas aquellas teorías personales definidas como las creencias, valores, entendimientos y asunciones que un profesor construye y se materializan en su práctica pedagógica, tal y como las identifica Crookes (2009). Estos elementos se encargan además de constituir las formas en que los docentes encuentran sentido a su experiencia profesional.

El tercer componente denominado las habilidades profesionales del docente, se define como todas las habilidades que le permiten de manera práctica realizar la labor docente. En el conocimiento de la asignatura, se incluye además la pregunta por lo didáctico, su capacidad reflexiva para tomar decisiones y habilidades sociales. Éstas permiten al docente la comunicación y cooperación en el contexto escolar no sólo con sus estudiantes, sino además con sus colegas, padres de familia u otros participantes de la comunidad académica. Finalmente el autor introduce el concepto de habilidades facilitadoras para puntualizar aquellas destrezas que le facilitan a un maestro un desarrollo profesional a lo largo de su carrera. Dentro de éstas se encuentran habilidades de lectura profesional, de presentación, de investigación y de teorización para mejorar su práctica.

El cuarto componente, actitudes y sentimientos acerca de la labor docente, implica las formas de pensar y las inclinaciones para actuar de cierta manera con respecto a la visión del lenguaje, la forma en que se aceptan las metas y propósitos educativos, la mirada sobre qué es la enseñanza y, por último, cómo se entiende y concibe la estructura de la escuela. En general los docentes construyen y demuestran actitudes y sentimientos hacia estas dimensiones con base en sus propias experiencias y la naturaleza cambiante de los procesos sociales.

Educación se erige como el quinto elemento de este modelo. Desde la pregunta "saber qué es la educación”, los docentes encuentran explicaciones que deben llevarlos a enfatizar en sus procesos los siguientes aspectos: el desarrollo de un aprendiz integral en términos de su conocimiento, sus habilidades y actitudes; la aplicación de los objetivos generales y específicos que expresa el currículo en toda la escuela y la preparación para aprender para la vida.

El último componente de este modelo se centra en el cambio y aprendizaje del docente, quien en el marco de una sociedad en constante transformación debe estar preparado y ser susceptible a cuestionar, a retar los modelos establecidos y a buscar el cambio. Estos procesos de cambio se presentan en las distintas esferas de la vida tales como en lo político, lo tecnológico, lo ambiental y, por su puesto, lo personal. En síntesis, el individuo está sujeto a estos cambios en estas esferas así como los currículos que él mismo como educador materializa a diario.

\section{El desarrollo profesional visto por académicos colombianos}

Si bien la formación permanente de docentes de inglés ha tenido una tradición en el país, pues solamente en los últimos 20 años se han publicado trabajos locales que recogen planteamientos teóricos al respecto. Es importante destacar que un creciente número de académicos colombianos coinciden en apreciaciones conceptuales sobre el desarrollo de los 
docentes de inglés en ejercicio enmarcados dentro de la estrategia de desarrollo profesional. Estos trabajos son el fruto de investigaciones que articulan el conocimiento internacional sobre el tema con los hallazgos particulares, produciendo conocimiento local pertinente a nuestro contexto.

Un importante marco de referencia para Colombia se halla en la propuesta que planteó para Colombia un grupo de docentes participantes en el proyecto Colombian Framework for English -COFE- (Aparicio et al., 1995). Las elaboraciones teóricas iniciales partieron de los postulados de Freeman (The descriptive model of teaching: the constituents, 1989); O'Brien (The E.R.O.T.I Model: a teacher training planning guide, 1981)5; y Wallace (The Reflective Model, 1991). Sus planteamientos bien pueden considerarse como un desarrollo en el área, que recogió modelos epistemológicos y planteó un marco conceptual pertinente y adecuado, en la perspectiva de responder a las características del país. La propuesta COFE, al igual que la propuesta de Richards \& Farrell (2005), se preocupa por varias dimensiones de la formación de un docente, resalta lo subjetivo y la reflexión como ejes para la construcción y el crecimiento profesional.

El primer componente de este marco conceptual presentado por el proyecto COFE, los constituyentes de la formación docente (Freeman, 1989), incluye el conocimiento, las habilidades, las actitudes y el concepto de conciencia. El primero se refiere al conocimiento de lo que el docente debe enseñar, a quién se le enseña y dónde. Estos componentes sitúan así, la enseñanza en un contexto socio-cultural en el que se localizan unos actores pedagógicos que actualizan y contribuyen a construir o destruir los conocimientos recibidos por el docente como producto de su formación profesional y como resultado de su praxis.

En concordancia con Freeman (1989), la propuesta colombiana inicial incluye los cuatro aspectos constitutivos de la enseñanza y los cuales

5 La sigla del modelo E.R.O.T.I. corresponde a Experience (experiencia), Rationale (fundamentación), Observation (observación), Trial (prueba), Integration (integración). se definieron en la sección introductoria de este artículo. Igualmente, la concepción local de desarrollo profesional para docentes en ejercicio incluye los procedimientos del proceso de la educación docente: experiencia, fundamentación, observación, prueba e integración (O’Brien, 1981). En este orden de ideas para secuenciar el proceso de formación docente, se deben poner en interacción estos elementos. Se ubica la fundamentación en el centro de los otros elementos y se busca que haya un punto de entrada al proceso desde tres de sus elementos (experiencia, observación y fundamentación) de acuerdo con las necesidades de los docentes. Por ejemplo, si se quiere integrar una nueva iniciativa pedagógica en una clase, se recomienda que se inicie con una experiencia directa en un rol de aprendiz. También se puede acceder a estudiar esa iniciativa desde un proceso de observación de pares. De igual forma el docente puede estudiar la fundamentación, la razón de ser que hace que esta nueva iniciativa sea valiosa. Posterior a alguno de los anteriores procesos -experiencia, observación o fundamentación- el docente puede acceder al siguiente estadio, el de prueba, haciendo una aplicación en su contexto educativo, para así llegar al último estadio de integración en el programa o currículo.

En el trasfondo de este proceso de la formación de los docentes, se encuentra el concepto de toma de decisiones. El acceso a cada uno de los elementos del modelo de O’Brien implica un proceso de toma de decisión en cada maestro y en él también tiene lugar el ejercicio de reflexión (Calderhead, 1987). El Proyecto COFE integra entonces el concepto de reflexión desde la propuesta de Wallace (1991) y su modelo de desarrollo profesional. Este requiere el desarrollo de una conciencia inquisitiva frente a la enseñanza y los contextos en los que ésta sucede. Para lograr esto, se atiende al conocimiento profesional desde la perspectiva científica y desde la perspectiva de la experiencia. El conocimiento profesional proviene entonces de dos fuentes: a) el que se recibe (relacionado con hechos y teorías, entre otros) y b) el que se construye (con base en la experiencia y el conocimiento experiencial). 
En conclusión, esta propuesta local inicial para el desarrollo profesional de los docentes en ejercicio recoge varios de los aspectos que hacen parte de los modelos internacionales descritos anteriormente en este artículo. Sin embargo, se nota una iniciativa por elaborar una propuesta más integradora de todos los procesos que subyacen a la compleja tarea de la formación y desarrollo profesional. Como enuncian Aparicio et al. (1995), el modelo está sujeto a las limitaciones impuestas por los contextos e incluso por los individuos que lo deben materializar.

A partir del año 2000 se nota un nuevo interés de académicos locales por comprender las dinámicas del desarrollo profesional de los docentes de inglés en ejercicio. Al respecto, los estudios realizados sobre al desarrollo profesional de docentes en el área del inglés como lengua extranjera en Colombia, parten de investigaciones que revelan las condiciones y expectativas que tienen los maestros. Las investigaciones adelantadas por González et al. (2001) y González (2003) han identificado las percepciones de los docentes como educadores en esta área y cómo estas concepciones se han relacionado con sus expectativas y respecto a la formación continua que deberían tener para realizar su labor. Igualmente, estos estudios han revelado cuáles son las necesidades de los profesores en cuanto a su preparación continua y por ende han señalado implicaciones directas para programas de profesionalización.

Los hallazgos en estos estudios revelan que las condiciones económicas y sociales de las comunidades en las cuales laboran los docentes, así como las de ellos mismos en cuanto a sus aspectos laborales, tienden en muchos casos a causar desmotivación en la realización de su labor. Demasiado trabajo y la falta de autonomía, al igual que de recursos, surgen como limitantes para los maestros. De la misma manera, los docentes perciben que tienen baja competencia en la lengua extranjera, en especial aquellos quienes han tenido que asumir el reto de enseñar la lengua en el nivel de básica primaria sin tener la preparación para realizar esta labor (Cárdenas, 2001; Valencia, 2006; Cadavid et al., 2004).

Los docentes de inglés en ejercicio buscan PDP que apunten a necesidades en sus dimensiones como trabajadores, aprendices e instructores (González, 2003). El primero se refiere a la necesidad de que los programas promuevan el reconocimiento laboral de los docentes, bien sea mediante la consecución de créditos académicos que se conviertan en estímulos salariales o en el otorgamiento de mejores condiciones para su trabajo tales como más recursos, menos horas de clase o menos estudiantes por curso. En otras palabras, los docentes esperan que los programas transformen sus condiciones laborales y les den la oportunidad de compartir lo aprendido con otros colegas. El docente como aprendiz es una dimensión que busca que los programas vayan más allá del saber técnico y apoyen el desarrollo del conocimiento en áreas más amplias del saber específico como el manejo de problemas en el aula, la enseñanza de valores, la conexión entre la vida académica y la realización personal propia y de sus alumnos. La tercera dimensión apunta al desarrollo de las competencias de enseñanza y de conocimientos profesionales. Esta es la dimensión más abordada en los PDP tradicionales y a menudo deja por fuera las otras dos.

Adicionalmente, el estudio reportado en González (2003) encontró que para los docentes de colegios públicos y privados sí existen necesidades diferentes que deben abordarse con programas diferentes. Esta diferencia se hace mucho más evidente en el nivel de competencia lingüística tanto para mantenerla como para mejorarla, ya que la mayoría de docentes que tienen título universitario y que laboran en instituciones públicas reportan una pérdida significativa de sus habilidades en inglés a lo largo de su experiencia docente (González, 1995). Los docentes advierten sobre la importancia de ofrecer programas que promuevan el desarrollo de sus habilidades lingüísticas en inglés.

Lo anterior se relaciona con aspectos que se han determinado como fundamentales al abordar las expectativas de los profesores en cuanto a su participación en programas de profesionalización. En primer lugar, los docentes consideran necesario diseñar programas en los cuales se trabaje con los maestros desde sus realidades sociales y culturales. Los programas deben, entonces, conocer y valorar 
las particularidades de las comunidades de donde provienen los maestros y deben ser coherentes con sus necesidades y expectativas. En este sentido, los programas propuestos deberían responder a la necesidad de apoyo que sienten los profesores al tener que enfrentar condiciones sociales y económicas críticas en sus comunidades, al igual que en sus factores laborales. En segundo lugar, los docentes esperan contar con cursos de formación continuada en los cuales puedan tener opciones variadas para aumentar su manejo y conocimiento de la lengua extranjera. La posibilidad de contar con tutores que posean una vasta experiencia y conocimiento en la enseñanza de la lengua extranjera, también ha sido señalada como una condición ideal en los cursos de profesionalización. De esta forma, se espera participar en programas que puedan responder a las diversas necesidades de todos aquellos que los cursan y que pertenecen a diferentes contextos y realidades. Finalmente, los docentes consideran valioso participar en programas en los cuales sus tutores posean experiencia sólida en el área de investigación (González, 2000) y también manifiestan gusto por aprender respecto a investigación y llevar a cabo esta clase de trabajo en sus contextos escolares.

Un elemento claramente definido en las elaboraciones teóricas colombianas sobre el desarrollo profesional de los docentes en ejercicio es la necesidad de que se incluya la investigación como un componente de base en cualquier programa que se proponga. Los PDP en Colombia han incorporado este componente en los últimos años, como lo expresan González (2007, 2009); Cárdenas (2000, 2003, 2004, 2006); Malagón y Cárdenas (2007) y Cárdenas y Nieto (2007). Al describir sus experiencias en el liderazgo de cursos de actualización con docentes del distrito de Bogotá, Cárdenas señala que "la investigación en el aula se ha convertido en un medio para promover el pensamiento crítico sobre nuestras propias prácticas pedagógicas y evaluar, cambiar y crecer profesionalmente" (2000, p. 16). Es importante anotar entonces que gracias a la centralidad que ha adquirido la investigación, ésta se ha convertido en un componente más de los modelos de desarrollo profesional en el país.
Docentes de universidades colombianas argumentan que es urgente formar un profesor que sea un actor más que un receptor pasivo (González, 2007; Álvarez, 2007; Quintero et al., 2003; Usma, 2009). Esta necesidad se hace más pertinente aún en su formación profesional, ya que es ahí donde se enfrentan los retos de la profesión. La forma de alcanzar esta habilidad es mediante la toma de decisiones y la participación en proyectos de investigación que le permitan construir conocimiento (Freeman, 1998). Una de las mejores alternativas para desarrollar la autonomía y definir los aspectos sobre los cuales los docentes quieren y pueden trabajar son los grupos de estudio (Álvarez Aguirre \& Sánchez Prada, 2005; Sierra, 2007; Cárdenas \& Nieto, 2007). Sierra (2007) recomienda esta estrategia en la medida en que empoderar a los docentes, desarrolla actitudes democráticas y de iniciativa, posibilitando la aparición de líderes académicos entre los mismos docentes. Es importante resaltar que algunas universidades han acogido posturas teóricas que valoran la construcción de conocimiento local (Canagarajah, 2005) bajo la mirada de concepciones post estructuralistas de la lengua y de la enseñanza (Kumaravadivelu, 1994, 2003, 2006). Bajo estas miradas integrales se mira al docente de inglés como usuario de la lengua, como analista de ésta, como pedagogo y como investigador de su práctica profesional.

Consecuentes con las directrices científicas acordadas por la comunidad internacional y nacional, el Ministerio de Educación Nacional de Colombia y las Secretarías de Educación han establecido tres pilares para los programas de formación permanente o PFPD: actualización, innovación e investigación (Secretaría de Educación de Bogotá D. C., 2006). Se espera que todos los programas propuestos por universidades e instituciones académicas respondan de manera clara, incluyendo estos tres aspectos, de manera que el docente pueda desarrollar su potencial de aprendizaje y de enseñanza.

Vemos así una tendencia creciente a que las universidades tengan propuestas académicas integrales para formar los docentes de inglés. Por ejemplo, Cárdenas (2004) comparte los resultados 
de una investigación llevada a cabo en programas de formación permanente para docentes (PFPD) en la Universidad Nacional, sede Bogotá. Estos programas incluyen un componente de desarrollo de la lengua inglesa, un módulo de metodología de enseñanza de la lengua extranjera y un componente de investigación acción en el aula. El estudio se centra en una caracterización de la labor investigativa llevada acabo por los docentes en el marco del programa. Esta labor es precisamente un elemento fundamental y plenamente reconocido en cuanto a los procesos reflexivos que involucra (Cochran-Smith \& Zeichner, 2005). A este respecto, el estudio reporta la transformación que comienzan a tener los maestros, pues ellos incorporan procesos de evaluación en su enseñanza a partir de su reflexión constante, lo cual les permite encontrar más conexiones entre la teoría y la práctica que subyace a su actividad pedagógica. En este sentido, la investigación lleva a los docentes a asumir actitudes más críticas, ya que cuestionan permanentemente su quehacer. Como resultado de esto, se da un proceso de toma de decisiones más coherente y el maestro agencia una perspectiva de cambio en sus prácticas. Los docentes amplían la visión de su trabajo al encontrar una nueva posibilidad de actualización y aprendizaje.

Otros estudios locales muestran la articulación entre las universidades y las necesidades de los docentes. Clavijo et al. (2004), al estudiar las innovaciones de un grupo de docentes en un curso de desarrollo profesional, reportan la inclusión de un componente reflexivo transversal a todo el trabajo de los profesores. Es así como desde las primeras acciones realizadas en el programa los docentes asumen una actitud crítica respecto a su quehacer, pues centran sus propuestas de trabajo pedagógico en las necesidades socio-económicas, afectivas y de aprendizaje de sus estudiantes. Posteriormente continúa la actitud reflexiva y dinámica de los docentes al implementar sus innovaciones. Asimismo, ponen en marcha sus planes pedagógicos y mantienen una evaluación y reflexión constante, lo que les permite realizar los ajustes necesarios para la buena marcha de su labor.

A su vez, McNulty y Quinchía (2007) describen la implementación de un programa de desarrollo profesional holístico para profesores de básica primaria, el cual en forma similar a los descritos anteriormente involucraba a los docentes en un enfoque reflexivo. El programa se centró en las necesidades de los participantes en cuanto a sus realidades educativas y la posibilidad de un currículo negociado con los maestros a partir de su proceso de aprendizaje. El programa buscaba el desarrollo de competencias en el manejo de la lengua extranjera y la reflexión pedagógica constante en los profesores. Los hallazgos basados en la evaluación del programa revelan que la mayoría de los profesores mostraron avances en sus habilidades comunicativas e iniciaron un proceso de reflexión respecto a su necesidad de continuar mejorando en este sentido. En forma similar, su confianza para utilizar el idioma, a pesar de sus limitaciones, se incrementó. De otro lado, los docentes también comenzaron a tomar riesgos en cuanto a la implementación de diversas metodologías que ellos mismos habían experimentado como estudiantes en el programa.

Finalmente, merece citarse el trabajo de Vergara et al. (2009), quienes dan cuenta de la experiencia de un grupo de profesores universitarios quienes tuvieron a su cargo el "Seminario de Investigación en el Aula", dirigido a un grupo de maestros participantes en el PDP de la Universidad del Valle. Las autoras identifican los logros y dificultades encontradas en trabajo con los maestros, quienes enfrentan problemáticas tanto en el aula universitaria como sus propias aulas escolares. De igual manera, identifican la incidencia que ejerce el trabajo desarrollado en el aula universitaria en la práctica profesional de los docentes participantes.

Los trabajos citados en esta sección representan una contribución significativa para la elaboración de propuestas académicas que apunten al desarrollo profesional de los docentes de inglés en Colombia. Los hallazgos reportados en estos estudios y su análisis deben ser parte de las revisiones bibliográficas de las propuestas elaboradas para Colombia, ya que parten de las necesidades de nuestros docentes y apuntan a la solución de problemas particulares a nuestro contexto. Su valor académico es altamente significativo y complementa las teorías producidas 
en contextos como Estados Unidos e Inglaterra que han orientado tradicionalmente la mayor parte de las propuestas para docentes de inglés en el país.

\section{Conclusiones e implicaciones}

La revisión bibliográfica de la literatura internacional y nacional nos permite concluir que los programas de desarrollo profesional que se propongan para los docentes de inglés en ejercicio en Colombia deben considerar los siguientes aspectos:

1. Tener como marco general la perspectiva del desarrollo y no la del entrenamiento.

2. Combinar los ámbitos de trabajo individual del docente y de grupos de docentes en el contexto institucional, incluyendo las perspectivas socioculturales de la enseñanza y del aprendizaje.

3. Basarse en la teoría post método para posibilitar la búsqueda de alternativas a cada contexto particular de enseñanza.

4. Promover la reflexión y la investigación en los contextos escolares y sociales viendo al docente como un constructor de conocimiento.

5. Promover la práctica significativa y el aprendizaje del inglés asociados a la adquisición y construcción de nuevos conocimientos.
Finalmente, es relevante agregar que las políticas educativas que han venido trazando los entes nacionales y regionales del país en los últimos años plantean grandes retos a las instituciones universitarias que ofrecen programas de lenguas extranjeras. Ellas han decidido ofrecer PDP con el fin de responder a los urgentes requerimientos inherentes a los planes de acción que se han planteado para garantizar la cobertura en la enseñanza del inglés en la educación preescolar, primaria y secundaria, y garantizar la calidad de la educación. Estas actividades plantean un gran reto para toda la comunidad académica, y en especial, para los formadores de docentes. Asumirlo por parte nuestra implicaría indagar en aspectos tales como el perfil y la preparación de los formadores de docentes o tutores, las concepciones epistemológicas que sustentan los PDP, la relación entre dichas concepciones y las prácticas pedagógicas que caracterizan dichos programas y la manera como éstas últimas inciden en los contextos escolares. Este artículo es apenas un aporte de lo que implicaría ese reto. Por eso esperamos motivar nuevas posibilidades de investigación y de creación de conocimiento local pertinentes a nuestros contextos educativos y motivar al desarrollo profesional de los docentes que trabajan en ellos. 


\section{Bibliografía}

Álvarez Aguirre, G. y Sánchez Prada, C. (2005). "Teachers in a public school engage in a study group to reach general agreements about a common approach to teaching english". PROFILE. Issues in a Teachers' professional developtment, vol.6, pp.119-132.

Álvarez, J. A. (2007). "Secuencias didácticas de los docentes de inglés del Departamento de Lenguas de la Universidad de la Salle: avances y reflexiones en torno a un proceso investigativo". Actualidades Pedagógicas, vol. 50, pp.117-137.

Álvarez, J. A. (2009). “An exploration of Colombian EFL teachers' knowledge base through teachers' reflection". Revista Linguagem \& Ensino, vol. 12, n. ${ }^{\circ} 1$, pp. $73-108$.

Aparicio, B., Benavides, J., Cardenas, M., Ochoa, J., Ospina, C., y Zuluaga, O. (1995). Learning to Teach. Teaching to Learn. COFE Project Working Document 5. London: Thames Valley University, pp. 3-100.

Borg, S. (2006). Teacher cognition and language education. New York: Continuum.

Cadavid, I., McNulty M. \& Quinchía, D. (2004). "Elementary English Language Instruction: Colombian Teachers' Classroom Practices". PROFILE. Issues in Teachers' Professional Development, vol.5, pp. 37-55.

Calderhead, J. (1987). "The quality of reflection in student teachers' professional Learning". European Journal of Teacher Education, vol.10, n. ${ }^{\circ} 3$, pp.269-278.

Canagarajah, S. (2005). Reclaiming the local in language policy and practice. (ed.). Mahwah, New Jersey: Erlbaum.

Cárdenas, M. L. (2000). "Action research by english Teachers: an option to make classroom research possible". Colombian Applied Linguistics Journal, vol. 2, n. ${ }^{\circ} 1$, pp.15-26.

Cárdenas, R. (2001). "Teaching English in primary: Are we ready for it?" HOW, A Colombian Journal for English Teachers, vol. 8, pp. 1-8.

Cárdenas, M. L. (2003). "Teacher researchers as writers: a way to sharing findings". Colombian Applied Linguistics Journal, vol. 5, n. ${ }^{\circ} 1$, pp.49-64.

Cárdenas, M. L. (2004). "Las investigaciones de los docentes de inglés en un programa de formación permanente". Íkala, Revista de Lenguaje y Cultura, vol. 9 , n. ${ }^{\circ} 15$, pp.105-137.
Cárdenas, M. L. (2006). PFPD “Red PROFILE" - Red de profesores de inglés como lengua extranjera para el fortalecimiento de sus competencias comunicativa, pedagógica e investigativa en la educación básica y media. Bogotá: Universidad Nacional de Colombia, Departamento de Lenguas. Mimeo.

Cárdenas, M. L. (2006a). "Bilingual Colombia. Are we ready for it? What is needed?" Proceedings of the 19th Annual English Australia Education Conference, Perth. Obtenido desde: [http://www.englishaustralia.com.au/index.cgi? $\mathrm{E}=$ hcatfuncs $\& \mathrm{PT}=\mathrm{s} 1 \& \mathrm{X}=$ get doc\&Lev1=pub_c07_07\&Lev2=c06_carde]

Cárdenas, M. L. y Nieto, M. C. (2007). "El papel de la investigación acción en el trabajo de grupos de estudio de docentes de inglés de educación básica y media del sector oficial". Simposio Internacional Investigación Acción y Educación en Contextos de Pobreza. Bogotá: Universidad de la Salle, mayo de 2007. Memorias CD.

Cárdenas, M. L. (2008). II Congresso Latino-Americano de Formação de Professores de Línguas. Encuentros y desencuentros en la formación de profesores de inglés en Colombia: una mirada a las políticas del 'Programa Nacional de Bilingüismo'. PUC-Rio de Janeiro, 27-29 de noviembre de 2008, p. 1-20.

Castro, R., Correa, M. y Lira, H. (2004). "Curriculum y evaluación”. Universidad del Bió-Bió. Chile. Consultado en Febrero 25 de 2009, obtenido desde: [http://zeus. dci.ubiobio.cl/ cidcie/guia/pag/pdf/presenta1.pdf ]

Clavijo, A., Guerrero, C H., Torres, C., Ramírez, L M., y Torres, N E. (2004). “Teachers Acting Critically upon the Currículum". Íkala, Revista de Lenguaje y Cultura, 9(15), 11-41.

Cochram-smith, M. \& Zeichner, K.M. (2005). Studying Teacher Education. The report of the AERA panel on research and teacher education. Mahwah, N.J y Londres: Lawrence Erlbaum Associates, Publishers.

Crookes, G. (2009). Values, philosophies, and beliefs in TESOL. New York: Cambridge University Press.

Dede, C., Ketelhut, D., Whitehouse, P., Breit, L. \& McCloskey, E. (2009). "A Research Agenda for Online Teacher Professional Development". Journal of Teacher Education, vol. 60, n. ${ }^{\circ} 1$, pp.8-19. Obtenido en Enero 10 de 2009 desde: [http://jte.sagepub.com/ cgi/reprint/60/1/8]. 
Desimone, L., Smith, T \& Ueno, K. (2006). “Are teachers who need sustained, content-focused professional development getting it?" An Administrator's Dilemma. Educational Administration Quarterly, vol. 42, n. $\left.{ }^{\circ} 2\right)$, pp.179- 215.

Díaz-Maggioli, G. (2003, a). Fulfilling the promise of professional development. IATEFL Newsletter Samples. Obtenido en Marzo 13 de 2008 desde: [http:// www.iatefl.org/content/newsletter/174.php].

Díaz-Maggioli, G. (2003, b). "Professional development for language teachers". EDO-FL 03-03. ERIC Digest.

Freeman, D. (1989). “Teacher training, development and decision-making. A model of teaching and related strategies for language teacher education". TESOL Quarterly, vol. 18, n. ${ }^{\circ} 3$, pp.501-514.

Freeman, D. (1998). Doing teacher research: From inquiry to understanding. New York: Heinle \& Heinle.

González, A. (1995). Language attrition and retention among non native speaker EFL teachers. Doctoral dissertation State University of New York at Stony Brook.

González, A. (2000). “The new millennium: more challenges for EFL teachers and teacher educators". Colombian Applied Linguistics Journal, vol. 2, n. ${ }^{\circ}$, pp. 5-14.

González, A. (2003). "Who is educating EFL teachers: A Qualitative Study of in-service in Colombia". Íkala, Revista de Lenguaje y Cultura. 8(14), 153-172.

González, A. (2007). "Professional development of EFL teachers in Colombia: Between colonial and local practices". Íkala, Revista de Lenguaje y Cultura, vol. 12, n. ${ }^{\circ} 18$, pp.309-332.

González, A. (2009). “On alternative and additional certifications in English language teaching: The case of Colombian EFL professional development". Íkala, Revista de Lenguaje y Cultura, vol. 14, n. ${ }^{\circ} 22$, pp.183-209.

González, A., Montoya, C. \& Sierra, N. (2001). "EFL Teachers Look at themselves: Could they Grow together?" HOW, A Colombian Journal for Teachers of English, vol.9, pp.29-33.

González, A., Montoya, C. \& Sierra, N. (2002). "What do EFL Teachers seek in Professional Development Programs?" Voices from the Teachers. Íkala, Revista de Lenguaje y Cultura, vol. 7, pp. 29-50.

González, A. \& QUINCHÍA, D. (2003). “Tomorrow’s EFL Teacher Educators". Colombian Applied Linguistics Journal, vol. 5, pp.86-99.
Grundy, S. (1998). Producto o praxis del currículo. Madrid: Morata JACKSON.

Imbernón, F. (1997). "La formación y el desarrollo profesional del profesorado". Hacia una nueva cultura profesional, pp. 37-60. Barcelona: Editorial Graó.

James, P. (2001). Teachers in action: Tasks for in-service language teacher education and development. Cambridge: Cambridge University Press.

Johnson, K. E. (2009). Second Language teacher education. A sociocultural perspective. New York: Routledge.

Kemmis, S. \& Mc Taggart, H. (1989). The action research planner. Victoria: Deakin University Press.

Knowles, M. (1984). Andragogy in Action. San Francisco: Jossey-Bass.

Kumaravadivelu, B. (1994). “The post-method condition: (E)merging strategies for second/foreign language teaching". TESOL Quarterly, vol. 28, n. ${ }^{\circ} 1$, pp. $27-$ 48 .

Kumaravadivelu, B. (2003). Beyond methods: macrostrategies for language teaching. New Haven: Yale University Press.

Kumaravadivelu, B. (2006). "TESOL Methods: changing tracks, challenging trends". TESOL Quarterly, vol. 40 , pp. $59-81$.

Loeb, S., Rouse, C. \& Shorris, A. (2007). "Introducing the Issue". Project Muse Scholarly Journals on line, vol. 17, n. ${ }^{\circ} 1$, pp. 3-13 Spring.

Malagón, L. \& Cárdenas, M. L. (2007). "La creación de grupos de estudio: Vivencias en el marco de un programa de formación permanente de docentes de inglés". Signum, vol. 10, n. ${ }^{\circ}$, pp.73-94.

MC Nulty, M. \& Quinchía, D. (2007). "Designing a holistic professional development program for elementary school english teachers in Colombia". PROFILE. Issues in Teachers' Professional Development, vol. 8, pp. 131-143.

Narváez, E. (2005). "La formación permanente de maestros desde la pedagogía por proyectos". Lenguaje, vol. 33, pp. 241-283.

O'Brient, T. (1981). The E-R-O-T-I model: a stimulating guide for teacher training. British Council ELT Documents, vol. 110, pp. 54 - 61.

Colombia. Ministerio de Educacion Nacional [MEN]. "Avances y proyección Programa Nacional de Bilingüismo (PNB)". Reunión Programa Nacional de Bilingüismo: encuentro con universidades. 23-24 agosto de 2007. Bogotá: Sin publicar. 
Pont, B. (2003). Beyond Rhetoric: Adult learning policies and practices. Paris: Organization for Economic and Cooperative Development.

Quintero, J., López, M. \& Zuluaga, C. (2003). “La investigación-acción mejora la planeación de clases en lengua extranjera". Íkala, Revista de Lenguaje y Cultura, vol. 8, n. ${ }^{\circ} 14$, pp. 39-56.

Richards, J. \& Farrell, T. (2005). Professional development for language teachers. Cambridge: Cambridge University Press.

Richards J. \& Lockhart, C. (1994). Reflective teaching in second language classrooms. Cambridge: Cambridge University Press.

Secretaría de Educación de Bogotá D. C. (2006). "Plan territorial de formación docente 2006-2007". Una oportunidad de transformación Pedagógica de la escuela. Bogotá: Serie Lineamientos de Política Alcaldía Mayor de Bogotá.

Shohamy, E. (2006). Language policy: Hidden agendas and new approaches. New York: Routledge.

Sierra, A.M. (2007). "The professional development of a facilitator through a study group". PROFILE. Issues in a Teachers' Professional Developtment, vol. 8, pp. 91-101.

Schon, D. (1987). Educating the Reflective Practitioner. San Francisco: Jossey Bass.
Stenhouse, L. (1987). "La investigación como la base de la enseñanza". Madrid: Ediciones Morata.

Stenhouse, L. (1984). "Investigación y desarrollo del currículo". Madrid: Ediciones Morata.

Usma, J. A. (2009). "Education and language policy in Colombia: Exploring processes of Inclusion, Exclusion, and Stratification in Times of Global Reform". PROFILE, Issues in Teachers' Professional Development, vol. 11, pp. 123-142.

Valencia, S. (2006). "Literacy practices, texts, and talk around texts: English language teaching developments in Colombia". Colombian Applied Linguistics Journal, vol. 8, pp. 7-37.

Vergara, O., Hernández, F. \& Cárdenas, R. (2009). “Classroom Research and Professional Development". PROFILE. Issues in a Teachers' Professional Developtment, vol. 11, pp. 169-191.

Wallace, M. (1991). Training foreign language teachers: A reflective approach. Cambridge: Cambridge University Press.

Woods, D. (1996). Teacher congition in language teaching: Beliefs, decision-making, and classroom practice. New York: Cambridge University Press.

Woodward, T. (1991). Models and metaphors in language teacher training. Loop input and other suggestions. Cambridge: Cambridge University Press. 
